\title{
An Experimental Study on Relationship Between Subliminal Emotion and Implicit Sequence Learning: Evidence from Eye Movements
}

\author{
Zhanglong $\mathrm{Lu}^{1}{ }^{1,}$, Jieqiong $\mathrm{Lin}^{1}$, Xiaoyu $\mathrm{Li}^{2}$ \\ ${ }^{1}$ School of Education, Zhengzhou University, Zhengzhou, China \\ ${ }^{2}$ Zhengzhou Central Hospital, Zhengzhou, China
}

Email address:

psylzl@zzu.edu.cn (Zhanglong Lu)

*Corresponding author

\section{To cite this article:}

Zhanglong Lu, Jieqiong Lin, Xiaoyu Li. An Experimental Study on Relationship Between Subliminal Emotion and Implicit Sequence Learning: Evidence from Eye Movements. International Journal of Psychological and Brain Sciences. Vol. 3, No. 1, 2018, pp. 1-6. doi: $10.11648 /$ j.ijpbs.20180301.11

Received: December 7, 2017; Accepted: December 18, 2017; Published: January 12, 2018

\begin{abstract}
The relationship between emotion and implicit sequence learning is one of the basic question in the fields of implicit learning. The current study adopted serial reaction time (SRT) task paradigm and eye-movement technology to explore the effect of emotion on implicit learning. A one-factor between-subjects experimental design was used, with subliminal emotion (positive vs. negative) as between-subjects variable. Dependent measure was saccadic reaction time. Results were showed as follows: (1) saccadic reaction time was less in positive emotion group than negative emotion group; (2) saccadic reaction time was decreasing with increasing blocks; (3) there was significant interaction between group and block, and simple effect analysis indicated that the saccadic reaction time in positive emotion group was decreasing with increasing blocks, while there was no significant block effect in negative emotion group; (4) the amount of implicit sequence leaning was significantly higher in positive emotion group than negative emotion group. The findings suggest that positive emotion promote implicit sequence learning.
\end{abstract}

Keywords: Consciousness, Emotion, Implicit Learning, Sequence Learning, Eye Movements

\section{Introduction}

Many of daily activities are full of emotion. Different emotion state has a different effect on cognitive activities, such as learning, memory, attention. The effects mainly include the interference effect of negative emotion and the promote effect of positive emotion. Although emotion has been widely studied, controversy remains regarding the relationship between emotion and implicit learning. Different researchers explain the results on account of different theories. Besides implicit and explicit learning depend on different various cognitive systems, which leads further researches on the relationship between emotion and implicit learning [1].

A study [2] using serial reaction time task demonstrated that depressive patients showed diminished learning effect which was only half of control group's. But emotional disorder was associated with various processing deficits, the results could not provide direct evidence for emotional effect on implicit learning. Thus, subsequent researchers used healthy people. Emotional pictures or facial expression pictures were usually adopted as emotion induction materials [3-4]. Apart from emotional pictures and music, some researches adopted idioms to explore the effect of semantic stimulus on implicit sequence learning. The results indicated the amount of implicit sequence learning was more in positive condition [4]. In order to discourage explicit learning during SRT task, Shang and colleagues [5] adopted positive and negative music pieces as emotion induction materials using a probabilistic sequence. Shang and colleagues [5] found that the implicit sequence learning was hindered by negative emotion. The above results indicated that positive emotion promoted the implicit sequence learning, while negative emotion had an interference effect.

In contrast, Some studies found that positive emotion weakened the implicit learning, whereas negative emotion had promotion effect. Pretz, Totz, \& Kaufman [6] adopted 
classical implicit learning paradigms-artificial grammar learning (AGL) task and SRT task to explore the effect of emotion on implicit learning, using pictures in IAPS to induce mood state. Pretz, Totz, \& Kaufman [6] found that negative emotion did not significantly improve implicit learning in the SRT task, but implicit learning was improved by negative emotion in the AGL task. It may due to different cognitive processes involved in two tasks which resulted in learning differently. However, some studies found that negative emotion facilitated implicit sequence learning. Huang [7] used pictures in CAPS, and adopted the way of masking stimuli to display emotion stimuli in order to prime participants with unconscious emotion. Huang aimed to find out how unconscious emotion influence implicit sequence learning. The results showed that negative emotion group performed better than positive emotion group regardless of reaction times or the amount of learning. A recent study, Dienes, Baddeley, \& Jansari [8] explored a method for quickly determining the rate of implicit learning, and compared participants in the positive emotion condition with those in the negative emotion condition. Dienes, Baddeley, \& Jansari [8] found that participants who was primed with negative emotion could learn more quickly than positive emotion's participants. The results indicated that negative emotion had promotion effect on implicit learning.

As can be seen from the literature, different researchers hold different views on the relationship between emotion and implicit sequence learning. Previous studies about how emotions influence implicit sequence learning have some disadvantages: Firstly, previous studies mainly adopt positive and negative visual or auditory materials to induce emotions, such as words, musics, videos which have emotional valence. However, there are individual and sexual difference existing in these materials, which may influence the effect of emotion induction. For example, facial expression pictures may mix other factors (i.e. appearance) and disturb the experimental outcomes. Montagne [9] found that female had a stronger ability to differentiate various facial expressions than male, and there were significantly individual difference for comprehensions of movies and musics. Wang [3] proposed that games may be a new way to trigger emotion, but games could only inspire limited emotions resulted from success or failure. Secondly, Mackie [10] proposed the "cognitive resource occupied theory", which suggested that negative and positive emotions would induce thinking activities unrelated with current cognitive tasks and occupied the cognitive resources ultimately. When the task needed for more cognitive resources, thinking activities induced by emotions would occupy more resources, due to the limitation of cognitive resources, the performance of current cognitive task decreased. Regardless of emotions, previous studies mostly primed emotions by supraliminal stimuli. However, this way could consume more cognitive resources to some extent and interfere with cognitive activities. Subliminal priming has advantages over supraliminal priming. Thirdly, most traditional researches about sequence learning asked participants to press buttons with their fingers. But key pressing was influenced by many factors. People in different age and physical conditions had significant differences. Helmuth [11] found that reaction time between patients and healthy people was various, and the young and the elderly also had distinct reaction speed. Therefore, there are limitations among traditional sequence learning using key pressing. Considering the limitations and shortcomings of previous studies, the present study improves them from the three aspects as follows: Firstly, the size of stimuli on the screen was manipulated to create approaching and receding stimuli, which induced negative and positive emotions separately. This method of emotion induction is confirmed effective and less individual differences. Hsee, Tu, Lu, and Ruan [12] varied letters's size to create three types of videos-approaching, receding and static. Participants rated their feelings from negative to positive on a 7-point scale (1-very negative; 7-very positive). The results revealed that individuals felt more negative (or less positive) when a stimulus was approaching rather than static or receding (approach aversion effect). The approach aversion effect appeared no matter whether the stimulus was initially positive or negative. Hsee, $\mathrm{Tu}, \mathrm{Lu}$, and Ruan [12] believed that humans have developed an ability to guard against approaching stimuli in order to survive. In addition, Williams, and Bargh [13] investigated the effects of spatial cues on people's affective responses and proposed that the activation of spatial closeness and distance should influence people's subjective experiences. In experiment 1, the spatial distance or closeness priming stimuli using a Cartesian-plane Coordinate System were presented to participants. Then participants read a book excerpt which was embarrassing and assessed the extent to which they liked the excerpt. The results showed that participants who were primed with spatial distance would like the embarrassing story more than those who were primed with spatial closeness. In experiment 2, participants were primed with spatial closeness or distance in the same way, but were asked to read a violent book excerpt and then rated their current positive and negative emotion. The results indicated that people in the sense of spatial distance condition reported less negative affect than people in the sense of spatial closeness condition. Overall, the results supported that motor and perceptual representations of physical distance affected people' feelings and thoughts. Spatial distance are inevitably associated with emotions because distance and safety have a hard-wired association. Secondly, subliminal affective priming has advantages over supraliminal affective priming [14]: the activation of subliminal affective priming to memory system is less; the function of subliminal affective priming is greater, and unconsciously perceived information puts more effect on emotions. What's more, Cheng and Gao [15] used commendatory and derogatory Chinese terms to explore the mechanism of emotion priming at different levels of consciousness. The results suggested that unconscious emotion priming was the outcome of automatic processing, while conscious emotion was aroused by specific cognitive processes but inhibited by additional cognitive processes. And Liao and Tao [16] found that there were two ways of 
unconscious emotion priming, and the ways were subliminal and masked presentation of emotional stimuli which had been proved in many areas of information processing. Therefore, in order to occupy the cognitive resource at the lowest, the current study adopted the way of presenting rapidly to induce implicit emotion priming. Thirdly, for the purpose of controlling the additional effect of key pressing, The current study adopted eye movement technology. Participants were asked to observe the location of target stimulus instead of key pressing. the saccadic response time was the indicator. Saccadic response time is saccadic latency, and it refers the interval between appearance of target stimulus and the first saccade which reveals the planning and executive function of saccade. Previous studies indicated that saccadic response time had the same function as traditional reaction time, and saccadic response time could reflect cognitive progresses of sequence learning effectively. Kinder, Rolfs and Kliegl [17] found that saccadic response time of regular sequence decreased gradually when participants completed a serial reaction time task, but it increased when a random sequence was presented. The results were in line with traditional reaction time, and indicated that saccadic response time was an effective indicator of measuring implicit learning.

The current study adopts implicit sequence learning paradigm and eye-movement technology to explore the effect of emotion on implicit learning. Experimental design is a one-factor between-subjects experimental design, and independent variable is subliminal emotion while dependent variable is saccadic reaction time. Additionally, subliminal negative and positive emotions was induced by quickly presenting approaching and receding stimuli.

\section{Materials and Methods}

\subsection{Ethics Statement}

The study was approved by the Ethics Committee of Zhengzhou University. An informed consent was obtained from all participants.

\subsection{Participants}

A total of 41 students of Zhengzhou university (27 female, 14 male) voluntarily participated in the experiment in return for a gift. The average of the participants was 20.81 years $(S D=1.49$ years). All had normal or corrected-to-normal vision, and all of them had not previously taken part in implicit sequence learning experiment. Participants were randomly assigned to one of two experimental conditions (negative emotion condition, $\mathrm{n}=21$; positive emotion condition, $\mathrm{n}=20$ ).

\subsection{Materials}

\subsubsection{Emotion Priming Materials}

A black square presented for $200 \mathrm{~ms}$ on a white background. In the negative emotion condition, the black square was initially small which occupied $10 \%$ of the screen, then gradually increased its size by $10 \%$ until it occupied $90 \%$ of the screen. Compared to the negative emotion condition, in the positive emotion condition the size of the black square changed in the reverse direction. The black square was initially large, occupying $90 \%$ of the screen, then gradually decreased its size by $10 \%$ until it occupied $10 \%$ of the screen. The emotion priming materials were presented before sequence task.

\subsubsection{Sequence Learning Materials}

Four black boxes were presented in a horizontal row at the center of the screen on a white background. A black dot appeared in one of the four locations (referred to as 1-4, from left to right). The target sequence consisted of 12 locations (4-1-3-2-1-2-4-3-1-4-2-3) and was created so that each location occurred three times and each possible transition (e.g. $1-2,1-3$, etc.) was never repeated. The random sequence also consisted of 12 locations that were created on the basis of the same parameters, but random sequence differed from the target sequence. Participants were given instructions to looking at the location of the black dot as quickly and accurately as possible.

\subsection{Experimental Design}

A one-factor between-subjects experimental design was used, with subliminal emotion (positive vs. negative) as between-subjects variable. The dependent variable was the saccadic response time.

\subsection{Apparatus}

An SR Research (Mississauga, Ontario, Canada) EyeLink 1000 plus eye-tracking system recorded subjects' eye movements with a sampling rate of $1,000 \mathrm{~Hz}$, and with a chin-forehead rest. This eye tracker has a high spatial $\left(0.01^{\circ}\right.$ of visual angle) using pupil tracking and corneal reflection, The materials was displayed on a 19-in. Dell monitor with a refresh rate of $75 \mathrm{~Hz}$ (resolution $1,024 \times 768$ pixels), The viewing distance was approximately $60 \mathrm{~cm}$. Viewing was binocular and only right eye was tracked as permitted by the quality of the calibration for right eye. The experiment was run with Experiment Builder 1.10 software.

\subsection{Procedure}

\subsubsection{SRT Task}

Participants were seated in a silent room with normal ambient illumination. Participants were randomly assigned into positive or negative emotion groups. Participants were shown an array of four boxes at the center of the screen. A black dot appeared in one of the four locations (referred to as 1-4, from left to right). Participants were given instructions to look at the black dot as quickly as possible and made as few mistakes as possible, but participants did not know that black dot would appear in a repeating sequence. After participants had read the instruction, participants were calibrated with a standard 5-point grid for both eyes. Calibration was applied at the beginning of every session. The experimenter checked the 
accuracy of the calibration at any time and recalibrate if necessary during the whole process of experiment. Especially when participant' eye was not detected by the eye tracker in the target location within 3,000 ms from target onset.

Each trial began with a central fixation cross. The fixation "+" appeared in the center of the screen for $250 \mathrm{~ms}$, followed by the emotion priming stimuli for $200 \mathrm{~ms}$, then the black dot was presented. In order to make sure that participants had understood the instructions, participants complected a practice block which consisted of 50 trials. Then completed the 12 learning blocks of 108 trials, for a total of 1296 trials. The Block 11 was a new random sequence, and the other blocks were the regular target sequence. And the initial position of each block was randomly selected, each session was separated by short breaks.

\subsubsection{Generation Task}

After the serial reaction time task portion of the experiment was finished, all participants conducted a generation task test [18]. The purpose of the generation task was to assess the extent to which participants could control their sequence knowledge strategically. Evaluating the level of explicit knowledge is important, that is because implicit learning and explicit learning are fundamentally different from each other and even supported by different systems.

Participants were informed that the order of target positions followed a predetermined sequence, in which every location is completely determined by the previous two locations. According to the Process Dissociation Procedure [19], under the inclusion condition participants were asked to generate a sequence that resembled the learning sequence as much as possible. Conversely, under the exclusion condition participants were asked to generate a new sequence, but participants had to try to avoid generating the sequential regularities of the learning sequence. In both cases, a restriction was implemented, namely that participants were forbidden from generating the same location twice or more in a row. Half of the participants did the inclusion test firstly, and then the exclusion test; and the other half participants completed the exclusion test firstly, and then the inclusion test.

\section{Results}

All analyses were conducted in Data Viewer version 1.11 and Statistical Product and Service Solutions (SPSS) version 21.0. Data from two participants who did not complete the whole experimental task were removed. According to performance in generation task test, eight explicit participants were excluded [20]. A total of 10 participants were excluded: five from the negative emotion condition and five from the positive emotion condition, leaving $n=31$ participants for the following analysis.

Figure 1 shows the mean saccadic response time in positive and negative emotion group obtained over the training phase. As can be seen in Figure 1, saccadic response time decreased gradually from Block 1 to Block 11 in positive emotion group. In Block 12, where a new random sequence was presented, saccadic response time increased in both groups and decreased again in Block 13 where the target sequence was resumed. To test this effect statistically, a 2 (Group: positive emotion, negative emotion) $\times 11$ (Block:1-11) mixed-design Analysis of Variance (ANOVA) was carried out with Block as a within-subjects variable and Group as a between-subjects variable. The results showed that the main effect of Group was significant, $\quad F(1,29)=13.53, \quad p<0.01, \quad \eta^{2}=0.32$, in which saccadic reaction time was less in positive emotion group $(491 \mathrm{~ms})$ than negative emotion group $(517 \mathrm{~ms})$. In addition, the main effect of Block was significant, $F(10,290)=9.98$, $p<0.01, \eta^{2}=0.26$, and saccadic reaction time was less with increasing blocks. And there was significant interaction between Group and Block, $F(10,290)=6.15, p<0.01, \eta^{2}=0.18$, and simple effect analysis indicated that the saccadic reaction time in positive emotion group was decreasing with increasing blocks, $F(10,140)=13.34, p<0.01, \eta^{2}=0.49$.

In order to determine whether participants had acquired knowledge of the learning sequence and whether the two groups had acquired the same or different amount of knowledge. The last three blocks (Block11、Block12 and Block13) performance were determined as sequence knowledge. The performance in the learning sequence blocks (average of Blocks 11 and 13) with the performance in the Block 12 where the sequence was random were compared. Sequence knowledge should improve participants' performance, if participants had acquired sequence knowledge, the performance should decline when the learning sequence was disappeared in Block 12. To obtain a measure of sequence learning [21], An ANOVA on saccadic reaction times with Group (positive emotion vs. negative emotion) as a between-subjects factor was performed. The results suggested that the main effect of group was significant, $F(1,29)=13.01$, $p<0.01, \eta^{2}=0.31$, the amount of implicit sequence learning was significantly higher in positive emotion group ( $37.8 \pm 16 \mathrm{~ms})$ than negative emotion group $(3.6 \pm 33 \mathrm{~ms})$.

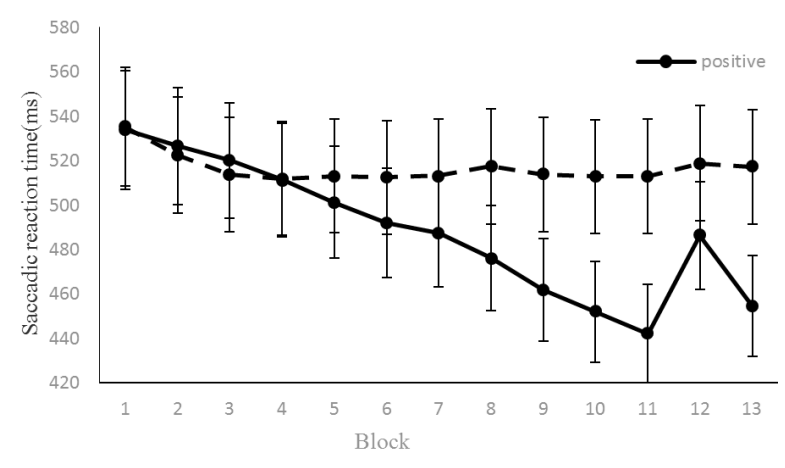

Figure 1. Saccadic reaction time in positive and negative emotion group.

\section{Discussion}

In the present study an observational version of serial reaction time task paradigm was designed to explore the effect of emotion on implicit sequence learning, in which participants merely looked at the target position rather than 
responding to it manually. The results showed saccadic response time decreased in the course of learning blocks and increased again when a new random sequence replaced the regular sequence on positive emotion group condition. The results revealed that saccadic response time exhibited the typical pattern normally seen in traditional manual response times. And saccadic reaction time was less in positive emotion group than negative emotion group, and the amount of implicit sequence leaning was significantly higher in positive emotion group than negative emotion group. The results suggested that positive emotion promoted implicit sequence learning, which was consistent with some previous researches [2-5]. It should be noted that with increasing blocks, there was no significant learning effect in negative emotion group. saccadic reaction time in negative emotion group decreased in the first four blocks and there was no increase in random sequence, it may be because of effects of familiarity and practice.

According to the broaden-and-build theory of emotion [22], positive emotion can broaden the scope of attention, occupy less cognitive resources and see the bigger picture, while negative emotion limits the scope of attention and processing. However, the relationship between implicit sequence learning and attention is unclear. Some studies revealed that implicit sequence learning was not affected by attention [23]. Recently $\mathrm{Fu}$ and $\mathrm{Fu}$ [24] proposed the dynamic demand hypothesis and believed that the more complex serial reaction time task was, the more attention was needed. Compared with artificial grammar task, serial reaction time task was relatively easier. and the present study adopted a deterministic sequence, participants may be easy to complete the serial reaction time task.

Besides, according to the affect-as-information hypothesis [5], negative emotion focuses on bottom-up processing and specific details which isn't beneficial to get the sequence structure. However, positive emotion is related to top-down and relation processing, and tends to integrate new information into previous knowledge to get integrative information. Thus, this assumption can explain our results to some extent. What's more, positive moods increase reliance on schemas and heuristics. "Negative moods narrow the focus of attention, increasing analytical processing, causal reasoning, and reliance on systematic processing" [25]. Within dual process theory, "implicit learning is a function of the implicit system, whose processing is unconscious, holistic, effortless, associative, and heuristic" [26]. So, positive emotion related to heuristic could promote implicit sequence learning. And task performance is influenced by motivation. Aarts [27] found that positive and negative emotion had different effects on unconscious goal seeking. Specifically, participants had stronger motivation and execution when positive emotion was presented with goals. We infer that positive emotion increase the level of motivation, and participants complete the task better. It remains unclear why there is no significant sequence learning effect of negative emotion, it may be because that negative emotion involves data-driven processing and focuses on specific items. But serial reaction time task has a sequence structure and each item is combined with other items to form the sequence, participants get the rule implicitly and benefit from the sequence. Consequently, participants primed with negative emotion only tends to response to item itself and can not learn the sequence. Additionally, previous study [7] showed that the effect of emotion on implicit learning was adjusted by emotion strength. It is useful to assess the valence strength of emotional stimuli for the future research.

\section{Conclusion}

To summarize, our findings indicate that implicit sequence learning may be influenced by subliminal emotion, the positive emotion promotes implicit sequence learning.

\section{References}

[1] Borbely-Ipkovich, E., Janacsek, K., Nemeth, D., \& Gond, X. The effect of negative mood and major depressive episode on working memory and implicit learning.

Neuropsychopharmacologia Hungarica, 2014, 16, 29-42.

[2] Naismith, S. L., Hickie, I. B., Ward, P. B., Scott, E., \& Little, C. Impaired implicit sequence learning in depression: a probe for frontostriatal dysfunction? Psychological Medicine, 2006, 36, 313-23.

[3] Wang, J. The implicit sequence learning about different types of emotional facial pictures. (Master dissertation, Zhejiang University). 2013.

[4] Yang, G. The research about implicit sequence learning of emotional and semantic stimulus. (Master dissertation, Shenyang Normal University). 2014.

[5] Shang, J., Fu, Q., Dienes, Z., Shao, C., \& Fu, X. Negative affect reduces performance in implicit sequence learning. Plos One, 2013, 8 (1):e54693.

[6] Pretz, J. E., Totz, K. S., \& Kaufman, S. B. The effects of mood, cognitive style, and cognitive ability on implicit learning. Learning \& Individual Differences, 2010, 20, 215-219.

[7] Huang, H. X. Implicit learning: the impacts of attention, goal, emotion and age. (Doctoral dissertation, Suzhou University). 2011.

[8] Dienes Z, Baddeley RJ, \& Jansari A. Rapidly measuring the speed of unconscious learning: amnesics learn quickly and happy people slowly. Plos One, 2012, 7, e33400.

[9] Montagne, B., Kessels, R. P., Frigerio, E., de Haan, E. H., \& Perrett, D. I. Sex differences in the perception of affective facial expressions: do men really lack emotional sensitivity? Cognitive Processing, 2005, 6, 136-141.

[10] Mackie, D. M., \& Worth, L. T. Processing deficits and the mediation of positive affect in persuasion. Journal of Personality \& Social Psychology, 1989, 57, 27-40.

[11] Helmuth, L. L., Mayr, U., \& Daum, I. Sequence learning in parkinson's disease: a comparison of spatial-attention and number-response sequences. Neuropsychologia, 2000, 38, 1443-1451.

[12] Hsee, C. K., Tu, Y., Lu, Z. Y., \& Ruan, B. Approach aversion: negative hedonic reactions toward approaching stimuli. Journal of Personality \& Social Psychology, 2014, 106, 699-712. 
[13] Williams, L. E., \& Bargh, J. A. Keeping one's distance: the influence of spatial distance cues onaffect and evaluation. Psychological Science, 2008, 19, 302-8.

[14] Lv, Y., Hu, W., Wu, G. L., \& Shen, D. L. An Experimental Study on Implicit and Explicit Learning Representations in a Serial Reaction Time Task. Psychological Science, 2008, 31, 770-773.

[15] Cheng, J. Q. \& Gao, X. P. The emotion priming under different levels of consciousness. Psychological Science, 2004, 27, 1506-1508.

[16] Liao, S. L. \& Tao, D. Q. New advances in studies about unconscious emotional priming. Psychological Science, 2004, 27, 701-704.

[17] Kinder, A., Rolfs, M., Kliegl, R. Sequence learning at optimal stimulus-response mapping: Evidence from a serial reaction time task. The quarterly journal of experimental psychology, 2008, 61, 203-209.

[18] Destrebecqz, A., \& Cleeremans, A. Can sequence learning be implicit? New evidence with the Process Dissociation Procedure. Psychonomic Bulletin \& Review, 2001, 8, 343-350.

[19] Jacoby, L. L. A process dissociation framework: separating automatic from intentional uses of memory. Journal of Memory \& Language, 1991, 30, 513-541.

[20] Lv, Y., Zhang, W. N., \& Shen, D. L. The priming effect of subliminal emotion face in different hedonic value: the evidence from ERP. Acta Psychologica Sinica, 2010, 42, 929-938.

[21] Nissen, M. J., \& Bullemer, P. Attentional requirements of learning: evidence from performance measures. Cognitive Psychology, 1987, 19, 1-32.

[22] Fredrickson, B. L., \& Branigan, C. Positive emotions broaden the scope of attention and thought-action repertoires. Cognition and Emotion, 2005, 19, 313-332.

[23] Lu, Z. L., Lv, Y., \& Bai, X. J. Attention load has no effect on implicit sequence learning: an eye movement study. Studies of psychology and behavior, 2011, 9(3), 214-218.

[24] Fu, Q. F. \& Fu, X. L. Relationship between Implicit Sequence Learning and Attention. Advances in psychological science, 2006, 14(6), 817-821.

[25] Bodenhausen, G. V., Kramer, G. P., \& Suesser, K. Happiness and stereotypic thinking in social judgment. Journal of Personality and Social Psychology, 1994, 66, 621-632.

[26] Pham, M. T. Emotion and rationality: A critical review and interpretation of empirical evidence. Review of General Psychology, 2007, 11, 155-178.

[27] Aarts, H., Custers, R., \& Veltkamp, M. Goal priming and the affective-motivational route to nonconscious goal pursuit. Social Cognition, 2008, 26, 555-577. 